\title{
Hair transplantation in patients with hair loss or scar deformity in the side hairline after midface- lifting surgery
}

\author{
Yang Seok Kim ${ }^{1}$, Young Cheon $\mathrm{Na}^{1}$, Jae Hyun Park ${ }^{2}$ \\ ${ }^{1}$ Department of Plastic and Reconstructive Surgery, Wonkwang University School of Medicine, Iksan; ${ }^{2}$ Dana Plastic Surgery Clinic, Seoul, \\ Korea
}

Background Successful aesthetic plastic surgery is devoid of both unsightly scarring and postoperative disfigurement. Patients undergoing midface-lifting surgery are very often disconcerted by an altered side hairline, including sideburns, despite considerable amelioration of facial wrinkles. This study was conducted to identify an effective means of approaching an altered hairline and the unavoidable scarring arising from midface-lifting surgery.

Methods A total of 37 patients who underwent corrective surgery with hair transplantation for hair loss or scar deformity arising from midface-lifting surgery from June 2014 to June 2017, and were observed for more than 6 months thereafter, were enrolled in the study. Prior to corrective surgery, the patients were administered a multiple-choice survey regarding their dissatisfaction arising from midface-lifting surgery. Among the 37 patients, 24, 12, and one underwent donor harvesting by the strip method, non-shaven follicular unit extraction, and partial shaving follicular unit extraction, respectively. Additionally, 33 of the 37 patients underwent hair transplantation in the frontotemporal recess area along with hairline correction surgery. The average number of transplanted grafts was 1,025.

Results Surgery resulted in a natural and satisfactory appearance in all patients. The average patient and physician subjective satisfaction scores were 4.6 and 4.8, respectively. No adverse events such as folliculitis occurred.

Conclusions Side-hairline correction surgery by hair transplantation can be considered an effective method of realigning an altered hairline accompanied by scars following midfacelifting surgery.

Keywords Rhytidoplasty / Lifting / Hair follicle
Correspondence: Jae Hyun Park Dana Plastic Surgery Clinic, 606 Gangnam-daero, Gangnam-gu, Seoul 06038, Korea

Tel: +82-2-512-0922

Fax: +82-2-512-0942

E-mail: jay8384@naver.com
This article was presented at the 2018 5th International Meeting of Aesthetic Plastic Surgery, on April 7, 2018.

\section{INTRODUCTION}

Plastic surgery is a specialty involving cosmetic improvements, and excellent patient satisfaction scores are therefore expected. The surgeon must endeavor to provide not only substantial cos- metic enhancement, but also natural results with no detectable traces of surgery. To achieve this goal, surgery should produce neither alteration/disfigurement of a normal body structure nor scar tissue; at the very least, the incision should be concealed to be less conspicuous [1]. Therefore, when performing various 
types of facial plastic surgery, every effort is made to create hidden scars by making incisions on the scalp or hairline.

Nonsurgical scarless facial thread lift is also an option for minimizing scarring [2]. However, the procedures that ensure the best results, with the highest satisfaction rates, involve an incision in the temple area, with excisional removal of excess skin to pull the skin tight for facial rejuvenation. Such face-lifting surgery involves making an incision along the side hairline or on the temporal scalp for camouflage, inevitably producing an incision scar on the scalp, along with a hairline alteration.

Classic midface-lifting surgery creates a youthful facial contour by eliminating wrinkles and restoring midface fullness. This procedure targets signs of aging such as wrinkles, but leaves many patients unsatisfied due to a deformed hairline or noticeable scarring along the hairline.

The authors obtained very satisfactory results by restoring altered hairlines through hair transplantation, and herein report the outcomes of this procedure with a review of the literature.

\section{METHODS}

Institutional Review Board/Ethics Committee approval was obtained from Wonkwang University Hospital (WKIRB201810-SB-075).

A total of 37 patients who received corrective surgery with hair transplantation to repair hair loss or incision scars as complications of midface-lifting surgery from June 2014 to June 2017, and were observed for at least 6 months thereafter, were included in the study. Prior to surgery, a dissatisfaction survey was conducted to assess complaints concerning scars, hair loss, or alterations of the side hairline.

The patients' average age was 42 years (range, 30-65 years). The interval from midface-lifting surgery to hairline correction surgery ranged from 2 weeks to 9 years, with an average interval of 1.6 years. The strip method was used in 24 patients, nonshaven follicular unit extraction in 12 patients, and partial shaving follicular unit extraction in one patient. Of all 37 eligible patients, 33 underwent hair transplantation in the frontotemporal recess area concurrently with hairline correction, with an average of 1,025 grafts (range, 420-2,290 grafts). Patients with an observation period of $<6$ months or who underwent additional procedures for midface-lifting, laser treatment, or any other cosmetic surgery were excluded from the study.

\section{Satisfaction survey}

A satisfaction survey was administered to the patients $>6$ months after the procedure. Written informed consent was received from the patients before the survey. Medical charts and photographs were retrospectively reviewed. The patients and surgeon scored the results in terms of satisfaction using a 5-point Likert scale ( 1 point, very dissatisfied; 2 points, dissatisfied; 3 points, neither satisfied nor dissatisfied; 4 points, satisfied; 5 points, very satisfied).

The survey questions were as follows: (1) How satisfied are you overall with the surgical results? (2) How natural do you think the results look?

Patients were requested to limit their responses to the above inquiries to hairline alterations, traces of scars, and hair loss caused by midface-lifting surgery.

\section{Representative cases}

Case 1

A 38-year-old woman visited Dana Plastic Surgery Clinic (Seoul, Korea) with chief complaints of significant midface widening; posterior displacement of her sideburns, creating a bizarre appearance with the sideburn attached to the ear; and a wide forehead. All abnormalities had developed after a midface-lifting procedure that she had undergone 3 years previously. She received hair transplantation in the frontotemporal recess area along with side hairline correction and medial repositioning of the sideburns. In total, 2,016 grafts harvested by the strip method were transplanted (Fig. 1).

\section{Case 2}

A 41-year-old woman visited our clinic for correction of a series of complications following a midface-lifting procedure that she had undergone 2 years previously, including a notch-shaped scar in the supra-auricular area, loss of sideburns, a widened infratemple area, and an irregular and wide forehead. In total, 1,740 grafts harvested by the strip method were placed (Fig. 2).

\section{Case 3}

A 52-year-old woman visited our clinic with complaints of a notch-shaped scar in the supra-auricular area and an awkward appearance created by posterior repositioning of the sideburns in close proximity to the ear. She underwent hair transplant of 457 grafts using non-shaven follicular unit extraction (Fig. 3).

\section{RESULTS}

The patients' dissatisfaction with their previous surgical procedures is shown in Table 1, including the frequency of each item. Hair growth observed at 10 months postoperatively or later showed a natural appearance, and all patients were satisfied with the results of surgery. The average satisfaction score for the patients and doctors was 4.6 and 4.8 , respectively. Two patients 
Fig. 1. Midface-lifting surgery deformity correction for case 1

$(A, B)$ Preoperative photograph. $(C, D)$ Surgery design. The height of the forehead was reduced by $1 \mathrm{~cm}$ along with total hairline correction surgery including coverage of the frontotemporal recess, temporal peak creation, and sideburn anterior advancement. (E, F) One-year postoperative photograph.
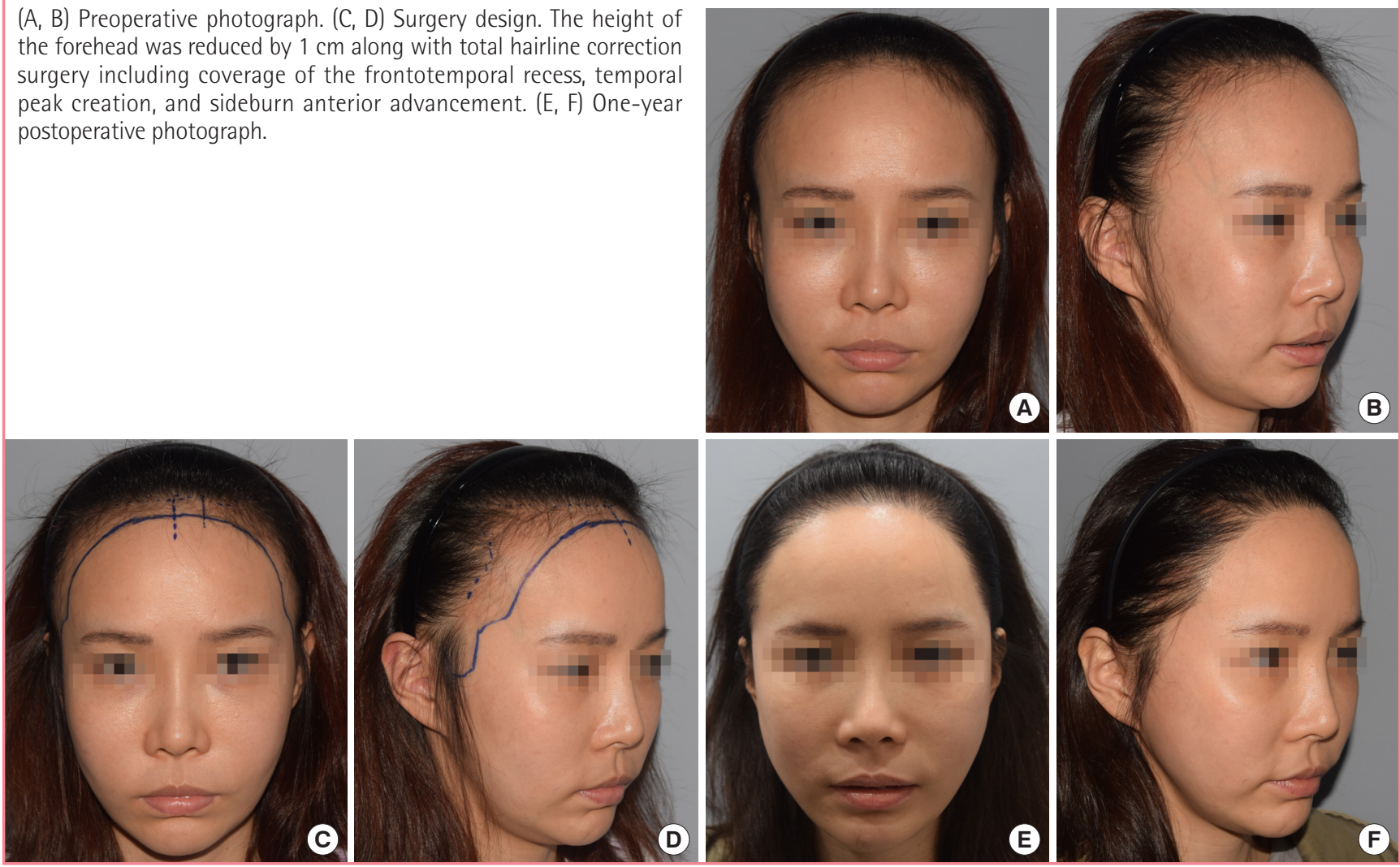

Fig. 2. Midface-lifting surgery deformity correction for case 2

(A-C) Preoperative photograph. (D-F) One-year postoperative photograph. Satisfactory results were achieved from overall hairline correction surgery accompanied by repair of the disfigurement and incision scars from midface-lifting surgery.
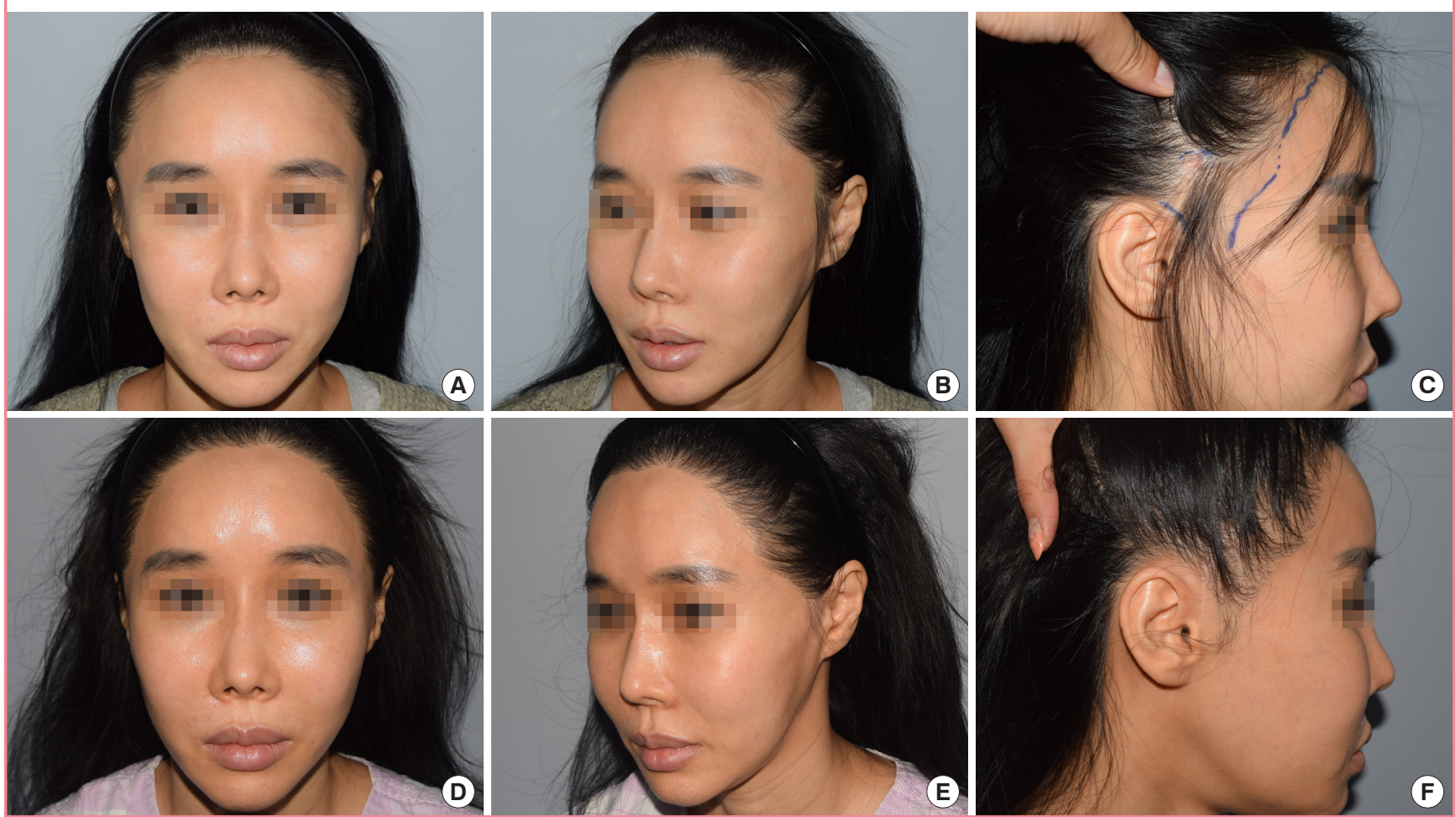


\section{Fig. 3. Midface-lifting surgery deformity correction for case 3}

(A) Preoperative view. (B) Immediate postoperative view. (C) One-year postoperative photograph. Natural-looking sideburns were newly formed at a more anterior position. Cicatricial alopecia was also successfully repaired through hair transplantation.
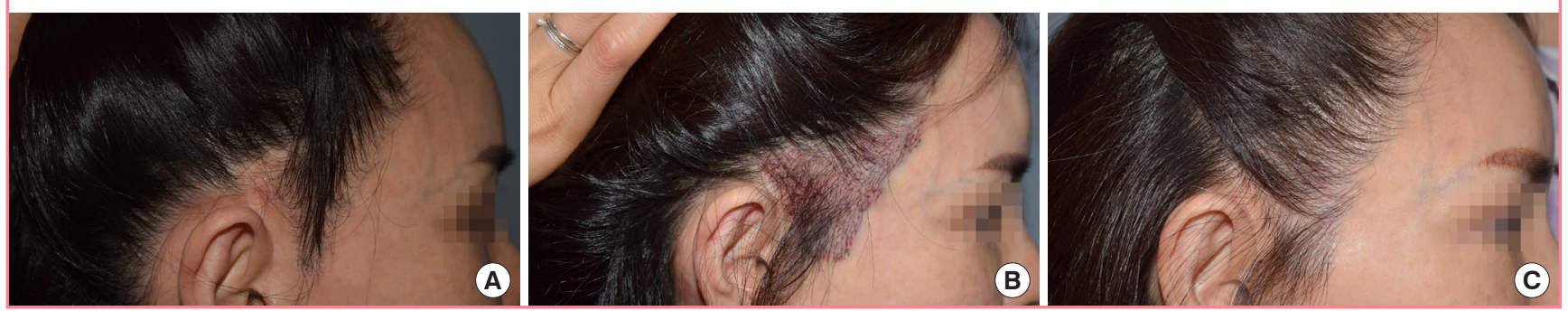

Table 1. Patients' dissatisfaction with prior midface-lifting surgery and problems detected upon physical examination $(n=37)$

\begin{tabular}{|c|c|c|c|}
\hline No. & Complaint & $\begin{array}{l}\text { Subjective } \\
\text { dissatisfaction } \\
\text { of patient }\end{array}$ & $\begin{array}{c}\text { Problems } \\
\text { detected upon } \\
\text { physical } \\
\text { examination }\end{array}$ \\
\hline 1 & $\begin{array}{l}\text { Alteration of hair direction of } \\
\text { sideburn }\end{array}$ & 2 & 6 \\
\hline 2 & Sideburn posterior displacement & 2 & 9 \\
\hline 3 & Loss of normal sideburn contour & 22 & 24 \\
\hline 4 & Bald spot scar in front of the ear & 21 & 26 \\
\hline 5 & $\begin{array}{l}\text { Conspicuous scars within the } \\
\text { temporal hairline }\end{array}$ & 3 & 17 \\
\hline 6 & Conspicuous scar in temporal area & 12 & 18 \\
\hline 7 & Widened midface & 18 & 22 \\
\hline \multirow[t]{2}{*}{8} & $\begin{array}{l}\text { Conspicuous posterior mastoid } \\
\text { area scar }\end{array}$ & 3 & 4 \\
\hline & $\begin{array}{l}\text { Limitation to certain hair styles due } \\
\text { to above complications }\end{array}$ & 31 & - \\
\hline
\end{tabular}

(5.4\%) underwent touch-up procedures for density enhancement, which required 120 and 279 grafts, respectively. Both patients were satisfied with the results. No adverse events such as folliculitis occurred.

\section{DISCUSSION}

Across all ages and cultures, people seek to maintain or regain youthfulness. Restoration of a youthful shape to the cheek, jowl, and neck requires a facelift [3]. There are many variations of midface-lifting surgery, which is considered one of the most popular procedures in the field of plastic surgery [3-7].

Lewis [8] reported that midface-lifting surgery may produce disfigurement of the sideburns and attempted to prevent this complication by making a temporal incision, which was reportedly incorporated in $>200$ patients with outstanding results. Marten [7] stated that conventional facelift surgery might lead to loss of the sideburns, a bald spot in front of the ear, and scars, along with a lateral sweep, malar crest, pulled mouth, and hollow eyes. To prevent these cosmetic problems, the author pointed out the significance of estimating the change in distance between the lateral orbit and the temple hairline after shifting the skin flap, referring to this estimation as the "temporal skin show." This distance has aesthetic importance and progressively increases with age. Marten [7] reported that the temporal skin show was about 4 to $5 \mathrm{~cm}$ or less in healthy and youthful-appearing patients, but greater in older patients or those with a history of a prior facelift. Marten [7] also commented that a temporal scalp incision that extends superiorly from the anteriorsuperior aspect of the ear is not appropriate for older patients or those undergoing a secondary or tertiary procedure. In contrast, the incision should be placed along the temporal hairline for patients with an expected temporal skin show of $>5 \mathrm{~cm}$.

However, midface-lifting surgery always leaves the patient with scars and distortion of the temporal or sideburn hairline. Additionally, many patients are dissatisfied about appearing less attractive due to a widened face, as indicated by the results of our survey.

The authors of the present report originally anticipated that the presence of unsightly scars or the absence of sideburns would ultimately limit the patients to certain hairstyles and thus bring them the most dissatisfaction. Unexpectedly, however, the survey showed that a very high proportion of patient dissatisfaction arose from the face appearing flattened and widened at the expense of wrinkle removal. Asians, who have a brachycephalic skeleton, tend to desire a more slender and smaller face $[8,9]$. Thus, posterior-superior displacement of the temporal hairline or failure to preserve the sideburns would result in retrogression, leaving patients even more discontent. When the infratemple or sideburn areas are widened, the face may appear to be enlarged and thus less appealing [10-12]. In the present study, the authors concealed scarring and recreated or relocated the sideburns or temporal hairline in an anterior-inferior direction with 
very satisfactory results.

Scars on the scalp are usually difficult to repair simply through scar revision techniques, but they can readily benefit from hair transplant surgery. Hairline correction surgery using hair grafting is especially helpful in treating patients whose hairline has been altered following face-lifting surgery. It shares the same basic techniques used in typical hair restoration or hairline correction procedures. However, it is not as simple as merely lowering the forehead or transplanting hair onto scars; in order to achieve satisfactory results, thorough consultation and meticulous surgical planning are mandatory for understanding the overall facial contour, condition, characteristics, and location of the incision scars, the degree of hairline alteration and the overall shape, and last but not the least, the patient's subjective complaints and demands.

When performing midface-lifting surgery, not only should rejuvenation of the facial contour and effacement of wrinkles be assessed, but the effect of surgery on the hairline should also be examined and properly addressed during the patient-doctor consultation to select the most adequate surgical regimen.

Hairline distortion or scarring after midface-lifting surgery can be effectively remedied through hairline correction surgery with hair grafting.

\section{NOTES}

\section{Conflict of interest}

No potential conflict of interest relevant to this article was reported.

\section{Ethical approval}

The study was approved by the Institutional Review Board of Wonkwang University Hospital (IRB No. WKIRB-201810SB-075) and performed in accordance with the principles of the Declaration of Helsinki. Written informed consents were obtained.

\section{Patient consent}

The patients provided written informed consent for the publication and the use of their images.

\section{Author contribution}

Data analysis and interpretation: Kim YS, Na YC. Study design, drafting and critical revision of the manuscript for important intellectual content: Park JH. Approval of final manuscript: all authors.

\section{ORCID}

Yang Seok Kim https://orcid.org/0000-0003-3161-7284

Jae Hyun Park https://orcid.org/0000-0002-9230-4102

\section{REFERENCES}

1. Hamra ST. Prevention and correction of the "face-lifted" appearance. Facial Plast Surg 2000;16:215-29.

2. Kang SH, Byun EJ, Kim HS. Vertical lifting: a new optimal thread lifting technique for Asians. Dermatol Surg 2017;43: 1263-70.

3. Mast BA. Advantages and limitations of the MACS lift for facial rejuvenation. Ann Plast Surg 2014;72:S139-43.

4. Warren RJ, Aston SJ, Mendelson BC. Face lift. Plast Reconstr Surg 2011;128:747e-764e.

5. Kamer FM, Frankel AS. SMAS rhytidectomy versus deep plane rhytidectomy: an objective comparison. Plast Reconstr Surg 1998;102:878-81.

6. Tonnard P, Verpaele A. The MACS-lift short scar rhytidectomy. Aesthet Surg J 2007;27:188-98.

7. Marten TJ. High SMAS facelift: combined single flap lifting of the jawline, cheek, and midface. Clin Plast Surg 2008;35: 569-603.

8. Lewis CM. Preservation of the female sideburn. Aesthetic Plast Surg 1984;8:91-6.

9. Park JH. Novel principles and techniques to create a natural design in female hairline correction surgery. Plast Reconstr Surg Glob Open 2016;3:e589.

10. Park JH. Side-hairline correction in Korean female patients. Plast Reconstr Surg Glob Open 2015;3:e336.

11. Park JH, Moh JS. Camouflaging the posterior zygomatic arch protrusion after zygoma reduction surgery. Aesthet Surg J 2012;32:661-4.

12. ParkJH. Masking the close eye appearance in the East Asian female population: infratemporal hairline reduction with hair grafting. Aesthetic Plast Surg 2016;40:921-5. 